\title{
A Study on Detection of COVID-19 in Lung CT Images Using Transfer Learning With Resnet Pre-Trained Model
}

\author{
Rajeswari R \\ Associate Professor, Department of Computer Applications, Bharathiar University, Coimbatore, India
}

Correspondence should be addressed to Rajeswari R; rajeswarilenin2711@gmail.com

Copyright (C) 2021 Made Rajeswari R et al. This is an open access article distributed under the Creative Commons Attribution License, which permits unrestricted use, distribution, and reproduction in any medium, provided the original work is properly cited.

\begin{abstract}
COVID-19 has impacted the lives of each and every person in the world. Diagnosis of COVID-19 using imaging systems can be integrated with the standard Reverse Transcription Polymerase Chain Reaction (RTPCR) test to perform the diagnosis more accurately. In this paper, transfer learning based method using a pre-trained deep neural network model viz., ResNet is proposed to classify COVID-19 computed tomography (CT) lung images. The pre-trained model is fine-tuned in order to make it learn the features specific to COVID-19 CT lung images. The proposed method is compared with the methods available in the literature. The results show that the proposed method is comparable to the existing methods.
\end{abstract}

KEYWORDS- COVID-19 CT image classification, transfer learning, ResNet pre-trained model

\section{INTRODUCTION}

Coronavirus Disease - 2019 (COVID-19) is caused by a new strain of coronavirus called 'SARS-CoV-2' which affects the respiratory tract of a person. It has symptoms such as sore throat, head \& body ache, cough, chills, breathing trouble, fever. Apart from causing the deaths of millions of people worldwide, COVID-19 pandemic has affected each and every sector of life including education, transport, public health, food supply, economy, sports and employment. According to the reports of World Health Oranization (WHO) there are 105,394,301 confirmed cases and 2,302,302 deaths globally [1] and 10,826,363 confirmed cases and 154,996 deaths in India [2] as on $7 \mathrm{Feb}$ 2021.

The Reverse Transcription Polymerase Chain Reaction (RT-PCR) test done using the nasopharyngeal sample is the standard test performed to analyze whether a person is infected with COVID-19 virus or not. The RT-PCR test is combined with the examination of chest X-Ray images or Computed Tomography (CT)-lung images to diagnose the severity and progression of the disease. RT-PCR tests are performed to confirm the COVID-19 infection and lung XRay or CT images help in pointing out the location of infection in lungs. COVID-19 virus most often affects the lungs causing damage to the linings of air sacs and inflammation. The inflammation can be visualized in chest $\mathrm{X}$-Ray images and CT scans as 'ground-glass opacity' (GGO). CT images are useful in detecting the GGO in early stages of COVID-19 [3]. Similarly, lung CT images have a high sensitivity i.e., they are able to detect more true positive cases more accurately [4].

Automatic detection of the presence of COVID-19 signs in lung CT images will help in diagnosing COVID-19 quickly and more accurately. In order to detect the presence of COVID-19 signs in images, feature extraction is necessary. Traditionally machine learning models proved to be effective in differentiating medical images of healthy and non-healthy people [5], [6]. These machine learning models depend on handcrafted features such as morphology, shape and texture. Deep learning models have various advantages in medical image classification. They have the capability to learn features automatically from medical images. They provide very high accuracy rates especially when the amount of data available for training is large. Recently deep learning models are widely used in processing medical images [7], [8]. Extraction of handcrafted features requires some domain expertise in order to extract the relevant information from medical images. Whereas deep learning models extract the features automatically that helps in medical image diagnosis. Training neural network models requires a huge amount of computing resources and time. Hence, recently transfer learning is widely used where a deep neural network model developed for a specific purpose is considered as the base model for another task. In this paper, the ResNet pretrained model [9] based transfer learning is utilized for classifying COVID-19 and non-COVID-19 lung CT images. Rest of this paper is organized as follows. Section II describes some existing works on classification of COVID-19 images. Section III elaborates the proposed method in detail. Section IV presents the results obtained using the proposed model. Section V gives the conclusion.

\section{RELATED WORK}

Diagnosis of COVID-19 is very much essential to control the spread of the disease. Imaging modalities such as $\mathrm{X}$ Ray and CT scan have been widely used in diagnostic systems. The typical steps associated with a diagnostic system are preprocessing, segmentation, feature extraction and classification. Machine learning techniques form an integral part of diagnostic systems and play a crucial role in diagnosis of COVID-19. With the advent of deep learning techniques, raw images are used to train the deep neural 
network. The trained model is then used for feature extraction and classification. Most of the deep neural networks used for diagnosis of COVID-19 use convolutional neural network $(\mathrm{CNN})$. A lot of research work has been carried out in the diagnosis of COVID-19. Literature survey and comparative study of various techniques proposed for COVID-19 diagnosis have also been carried out. Bhattacharya et al. have presented a survey on the usage of various image processing and deep learning techniques for diagnosis of COVID-19 [10]. They have elaborated on deep learning techniques, applications of deep learning in medical image processing and usage of deep learning for COVID-19 diagnosis. Jain et al. have implemented pre-trained models and have compared the results obtained for COVID-19 images [11]. The results are analyzed with those obtained from InceptionV3, Xception and ResNet. Their analysis shows that Xception based model gives better results compared to other models.

Various machine learning methods and deep learning methods are also used for diagnosis of COVID-19. Traditional machine learning methods require handcrafted features whereas deep learning methods learn the features automatically from the input given. Elaziz et al. have proposed a deep neural network based method for classification of COVID-19 X-Ray images [12]. They have extracted features using fractional multichannel Exponent Moments (FrMEMs). An optimization algorithm is used to select optimal features which are then used by k-Nearest Neighbour classifier. Zhang et al. have proposed a fusion of features extracted from $\mathrm{CNN}$ and 308 handcrafted features obtained from various statistical measures [13]. They have used support vector machine for classification. They have also used histogram equalization to improve the contrast of the image. Perumal et al. have proposed a deep learning based approach which uses fine-tuning of layers and Haralick features [14]. They have used three different models viz., VGG16, ResNet50 and InceptionV3 model to differentiate COVID-19 infected X-Ray images from normal images. They have performed histogram equalization, Wiener filtering and cropping to improve the quality of images. Abbas et al. have proposed a deep CNN based model called Decompose, Transfer and Compose (DeTraC) to recognize COVID-19 X-Ray images [15]. Their method uses steps including local deep feature extraction, class decomposition, training and fine-tuning. Gupta et al. have proposed InstaCovNet-19 deep neural network to classify normal, COVID-19 and pneumonia chest X-Ray images [16]. Each and every pre-trained model such as InceptionV3, MobileNetV2, ResNet101, NASNet and Xception extracts unique features from images which support classification. Hence, they have used all these models and fuse the features extracted by each model to classify the chest X-Ray images. Sahlol et al. have proposed an Inception based CNN model to extract features from COVID-19 X-Ray images [17]. The relevant features are then selected using Fractional-Order (FO) and Marine Predators Algorithm (MPA). Although optimization is executed to extract the features they are able to get good accuracy values. Arias-Londono et al. have used the pretrained Chexnet model to classify COVID-19 chest X-Ray images [18]. Chexnet model is based on CNN and is trained using medical X_Ray images. They have also compared their results with ResNet50, InceptionV3 and InceptionResNetV2 models. As the model has already learnt features specific to chest X-Ray images it uses the learnt information to perform classification of COVID-19 chest X-Ray images.

\section{COVID-19 LUNG CT IMAGE CLASSIFICATION USING RESNET}

Transfer learning makes use of the knowledge present in a pre-trained model for some other task [19]. It helps in reducing the training time and increasing the accuracy. The accuracy of deep CNN depends on the size of the training datasets. The more the number of instances in the training dataset, the greater accuracy is produced by deep CNN. But it takes a very long time to train a deep $\mathrm{CNN}$ with huge datasets. This drawback is overcome by transfer learning. Transfer learning is based on the facts that (1) CNN learns information hierarchically in layers and that (2) the initial layers learn generic information present in all images and the other layers learn specific information. A process called fine-tuning is performed to obtain transfer learning. The initial layers of the pre-trained models are frozen as they have the generic information learnt already. The model with initial frozen layers is trained using the images in the current context. This makes these layers learn specific information present in the images of present task. Transfer learning is carried out in two ways. In the first method, as mentioned the initial layers are frozen and only the other layers are trained on the present dataset. The network then classifies based on the learnt features. In the second method all the layers in the convolutional base are fixed, i.e., none of the layers are trained on the present dataset. The pre-learnt features are given as input to the classification layer. There are various pre-trained models supported by keras such as VGG16, Xception and ResNet [20]. In the present work, ResNet models are used for classifying COVID-19 lung CT images. The VGG model consists of repeated structures to extract complex features. The bottleneck layer of Xception models helps in reducing the computational requirement. In ResNet, the added layer has to learn only the residual and hence it is able to support large number of layers. Both these characteristics help in improving the accuracy as well as in reducing the computational time. Residual Network is the abbreviation for ResNet and was introduced by He et al. in 2015 [9]. ResNet is CNN architecture with a good performance even if the number of layers is more. This is achieved by using a 'skip connection' or 'shortcut connection' in the basic block of ResNet as shown in fig. 1. It can be seen from figure 1 that if 'skip connection' is used the output is $F(x)+x$ and if it is not used the output is $F(x)$. This connection helps in solving the vanishing gradient problem by making the gradient to pass through this connection.

In this work, three pre-trained models of ResNet namely, ResNet50, ResNet101 and ResNet152 are used for classification of lung CT images. The architectures of these three models are given in table I. The convolutional layer performs the convolution operation on the input using the kernels or filters and extracts features from the input. The max pooling layer downsamples the input and thus reduces the computational complexity and the time required for training the parameters of the model. The fully connected layer helps in classifying the input. 


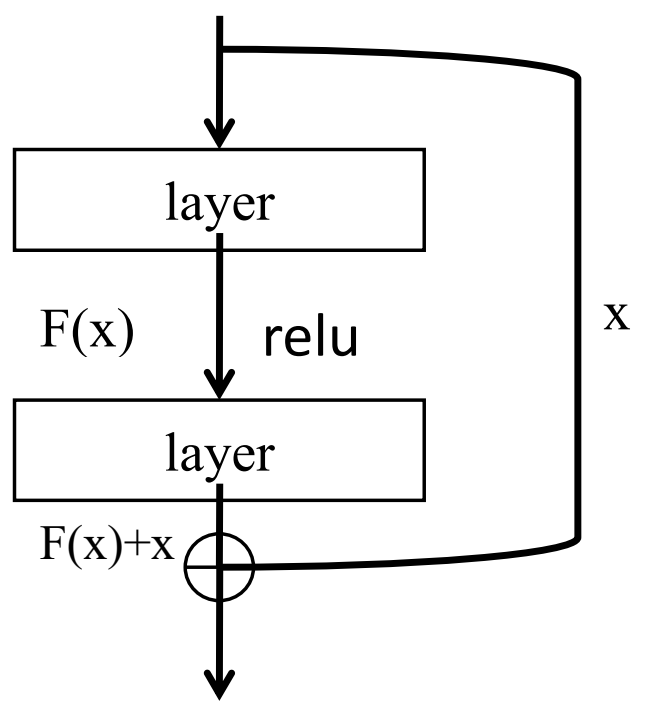

Fig. 1: Basic block of ResNet

The proposed method for classification of COVID-19 images is based on freezing the initial set of layers and finetuning the latter set of layers of ResNet model. The transfer learning based method for detecting COVID-19 in lung CT images is depicted in fig. 2. In the present work three different ResNet models viz., ResNet50, ResNet101 and ResNet152 are used. The first step is to create an instance of ResNet model. The images are resized to $224 \times 224 \times 3$ so that they can be given as input to the ResNet Model. Resizing all images to the same size introduces uniformity in the data used for training and testing. The next step is pre-processing which is carried out using the built-in module of ResNet model. The built-in preprocessing module of ResNet is used which consists of two stages. In the first stage, images in the red-green-blue format are converted to blue-green-red format. The blue-green-red format is recognized by the openCV package of python. In the second stage, every pixel is zero-centered with ImageNet database without scaling.

Table 1: Architecture of ResNet Models [9]

\begin{tabular}{|c|c|c|c|c|}
\hline $\begin{array}{l}\text { Layer } \\
\text { Name }\end{array}$ & $\begin{array}{l}\text { Output } \\
\text { Size }\end{array}$ & ResNet50 & ResNet101 & ResNet152 \\
\hline Input & & $224 \times 224 \times 3$ & $224 \times 224 \times 3$ & $224 \times 224 \times 3$ \\
\hline conv1 & $112 \times 112$ & $7 \times 7,64$ & $7 \times 7,64$ & $7 \times 7,64$ \\
\hline conv2_x & $56 \times 56$ & $\left.\begin{array}{l}1 \times 1,64 \\
3 \times 3, \\
3 \\
1 \times 1,256\end{array}\right\} 4$ & $\left.\begin{array}{l}1 \times 1,64 \\
3 \times 3,64 \\
3 \\
1 \times 1,256\end{array}\right\}$ & $\left.\begin{array}{l}1 \times 1,64 \\
3 \times 3,64 \\
1 \times 1,256\end{array}\right\}$ \\
\hline conv3_x & $28 \times 28$ & $\left.\begin{array}{l}1 \times 1,128 \\
3 \times 3,128 \\
4\end{array}\right\}$ & $\left.\begin{array}{l}1 \times 1,128 \\
3 \times 3,128 \\
4\end{array}\right\}$ & $\left.\begin{array}{l}1 \times 1,128 \\
3 \times 3,128 \\
1 \times 1,512\end{array}\right\} 8$ \\
\hline conv4_x & $14 \times 14$ & $\left.\begin{array}{l}1 \times 1,256 \\
3 \times 3,256 \\
1 \times 1,1024\end{array}\right\} 6$ & $\left.\begin{array}{l}1 \times 1,256 \\
3 \times 3,256 \\
1 \times 1,1024\end{array}\right\} 23$ & $\left.\begin{array}{l}1 \times 1,256 \\
3 \times 3,256 \\
1 \times 1,1024\end{array}\right\} 36$ \\
\hline conv5_x & $7 \times 7$ & $\left.\begin{array}{l}1 \times 1,512 \\
3 \times 3,512 \\
3 \\
1 \times 1,2048\end{array}\right\}$ & $\left.\begin{array}{l}1 \times 1,512 \\
3 \times 3,512 \\
3 \\
1 \times 1,2048\end{array}\right\}$ & $\left.\begin{array}{l}1 \times 1,512 \\
3 \times 3,512 \\
1 \times 1,2048\end{array}\right\} 3$ \\
\hline $\begin{array}{l}\text { fully } \\
\text { connected }\end{array}$ & & 1000 & 1000 & 1000 \\
\hline output & & 1x2classes & $1 \times 2$ classes & $1 \times 2$ classes \\
\hline
\end{tabular}

The ResNet model is used as the base and two layers are further added. The two layers added are fully connected layer with 1000 units and an output layer with two units. The fully connected layer helps in changing the domain of the data so that the classification layer works effectively. The default 'ReLU' function is used for the convolution and max pooling layers. The 'softmax' activation function is used for classification in the output layer. 'Softmax' function performs better compared to 'sigmoid' function for binary classification problems. Adam optimizer is used for updating the weights in the training stage. In order to avoid overfitting of data early stopping is used. Training is carried out with a drop out value of 0.5. These drop out layers along with the 'softmax' function helps in avoiding over-fitting of data. The cost-metrics used during training are cross entropy loss and accuracy. The hyperparameters used during the training stage of the ResNet model are summarized in table II. The ResNet models used in the present work have one input layer, five convolution layers, one fully connected layer and one output layer as given in table I. In the present work, the first four convolution layers and the first two sublayers of the fifth convolution layer is freezed. The frozen layers retain the weights already learnt by them. Fine-tuning is used in the present work. The third sublayer of the fifth convolution layer, fully connected layer and the output layer are unfreezed and trained on the COVID-19 CT lung image dataset. This helps in fine-tuning the constructed model using ResNet and learning the features specific to the COVID-19 CT lung images. The weights of the fine-tuned layers are updated based on the loss function and optimization algorithm used. The number of trainable and non-trainable parameters for the three fine-tuned ResNet models used in the present work is given in table III. This fine-tuning supports the usage of smaller number of images available in the COVID19 dataset to train a deep neural network.

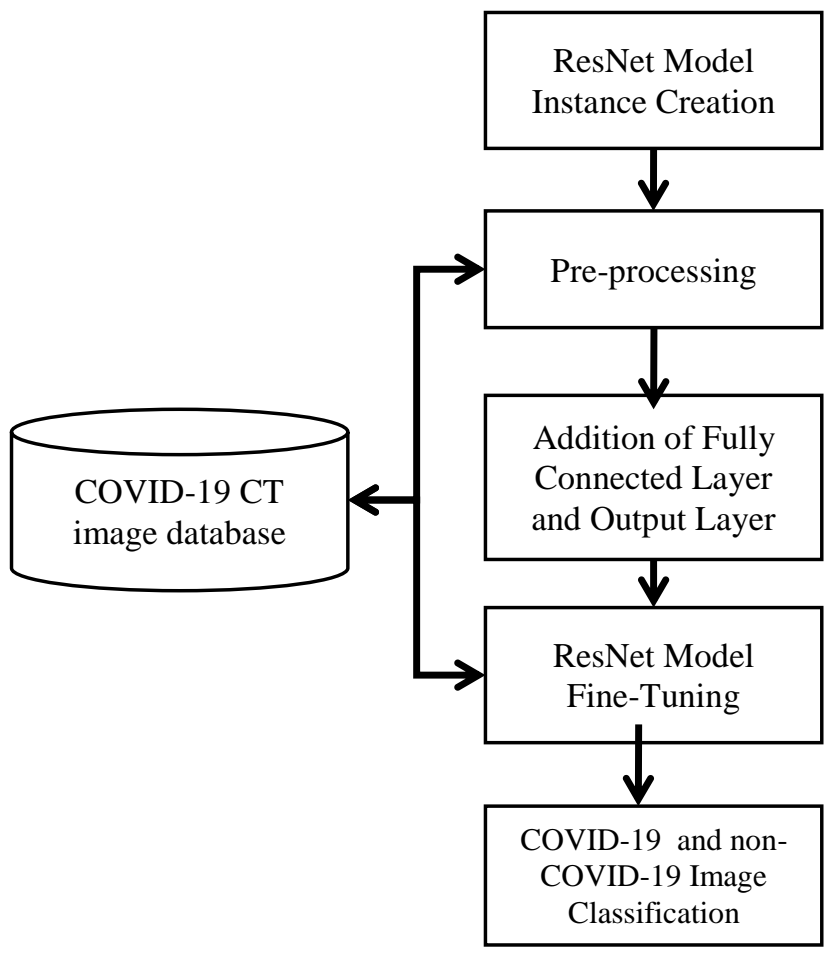

Fig. 2: Transfer Learning based classification of COVID-19 images using ResNet 
Table 2: Hyperparameters used in the pre-trained ResNet Model used in the present work

\begin{tabular}{|l|c|}
\hline \multicolumn{1}{|c|}{ Hyperparameter } & Value \\
\hline Stride & 1 \\
\hline Learning rate (default) & 0.001 \\
\hline Weight decay & 0.0001 \\
\hline Training epochs & 10 \\
\hline Batch size & 20 \\
\hline No. of units in fully connected layer & 1000 \\
\hline
\end{tabular}

Table 3: Trainable and Non-Trainable Parameters of FineTuned ResNet Models

\begin{tabular}{|l|r|r|}
\cline { 1 - 1 } No. of Parameters & Trainable & Non-Trainable \\
\cline { 1 - 1 } Models & & 10888386 \\
\cline { 1 - 2 } ResNet50 & 37916610 & 11164288 \\
\hline ResNet101 & 56934850 & 7858816 \\
\hline
\end{tabular}

\section{EXPERIMENTAL RESULTS}

Dataset consisting of lung CT images of SARS-CoV-2 infected patients and normal patients available in [21] is used in this work. The dataset consists of $1252 \mathrm{CT}$ images that are positive for COVID-19 and $1230 \mathrm{CT}$ images that are negative for COVID-19. The total number of CT images, ie. 2482 images were divided randomly into $90 \%$ for training and $10 \%$ for testing. Fig. 3 presents sample images which are COVID-19 positive and COVID-19 negative.
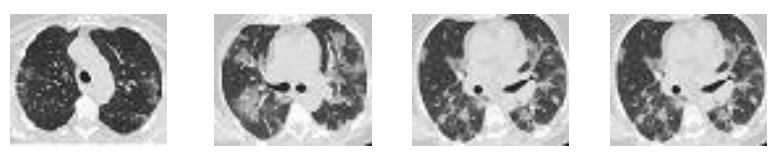

(a)
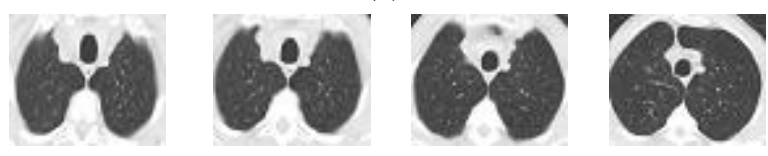

(b)

Fig. 3: Sample images (a) COVID-19 positive and (b) COVID-19 negative

The plots showing the accuracy values and loss during training and validation stages of all the three models after fine-tuning are presented in fig. 4. During training stage the number of epochs is 10 . It can be noted that the accuracy value of ResNet152 model is almost same right from the first epoch. Whereas its loss value slowly diminishes as the number of epochs increases. In the case of ResNet50 and ResNet101, the accuracy values start converging after $4^{\text {th }}$ epoch.

The accuracy values obtained during the training and testing stages of the three ResNet models are given in table
IV. The accuracy values before and after fine-tuning the ResNet models are given. It can be clearly seen that the ResNet models perform better after fine-tuning process and given higher accuracy values both during training and testing stages. Moreover, ResNet152 model gives better accuracy during testing stage. Although the number of layers is more in ResNet101, ResNet50 performs better during testing stage.

Table 4: Comparison of Accuracy values

\begin{tabular}{|l|c|c|c|c|}
\hline \multirow{2}{*}{ Models } & \multicolumn{2}{|c|}{$\begin{array}{c}\text { Training Accuracy } \\
(\%)\end{array}$} & \multicolumn{2}{c|}{$\begin{array}{c}\text { Testing } \\
\text { Accuracy }(\%)\end{array}$} \\
\cline { 2 - 5 } & $\begin{array}{c}\text { Before } \\
\text { Fine- } \\
\text { Tuning }\end{array}$ & $\begin{array}{c}\text { After } \\
\text { Fine- } \\
\text { Tuning }\end{array}$ & $\begin{array}{c}\text { Before } \\
\text { Fine- } \\
\text { Tuning }\end{array}$ & $\begin{array}{c}\text { After } \\
\text { Fine- } \\
\text { Tuning }\end{array}$ \\
\hline ResNet50 & 73.39 & $\mathbf{9 9 . 8 7}$ & 95.58 & $\mathbf{9 7 . 1 9}$ \\
\hline ResNet101 & 85.48 & $\mathbf{1 0 0 . 0 0}$ & 92.37 & $\mathbf{9 5 . 9 8}$ \\
\hline ResNet152 & 84.99 & $\mathbf{9 3 . 0 1}$ & 95.98 & $\mathbf{9 8 . 3 9}$ \\
\hline
\end{tabular}

The accuracy values obtained after fine-tuning the ResNet models are compared with the accuracy values obtained from various existing methods in the literature and the results are presented in table V. Most of the existing methods make use of chest X-Ray images. The proposed method using ResNet152 performs better compared to methods in [14] and [15]. The method proposed in [16] performs better compared to the proposed method. This can be attributed to the fact that it uses Chexnet pre-trained model which has already learnt features specific to chest XRay images. 
International Journal of Innovative Research in Computer Science \& Technology (IJIRCST)

ISSN: 2347-5552, Volume-9, Issue-2, March 2021

https://doi.org/10.21276/ijircst.2021.9.2.5

Article ID IRP1133, Pages 28-34
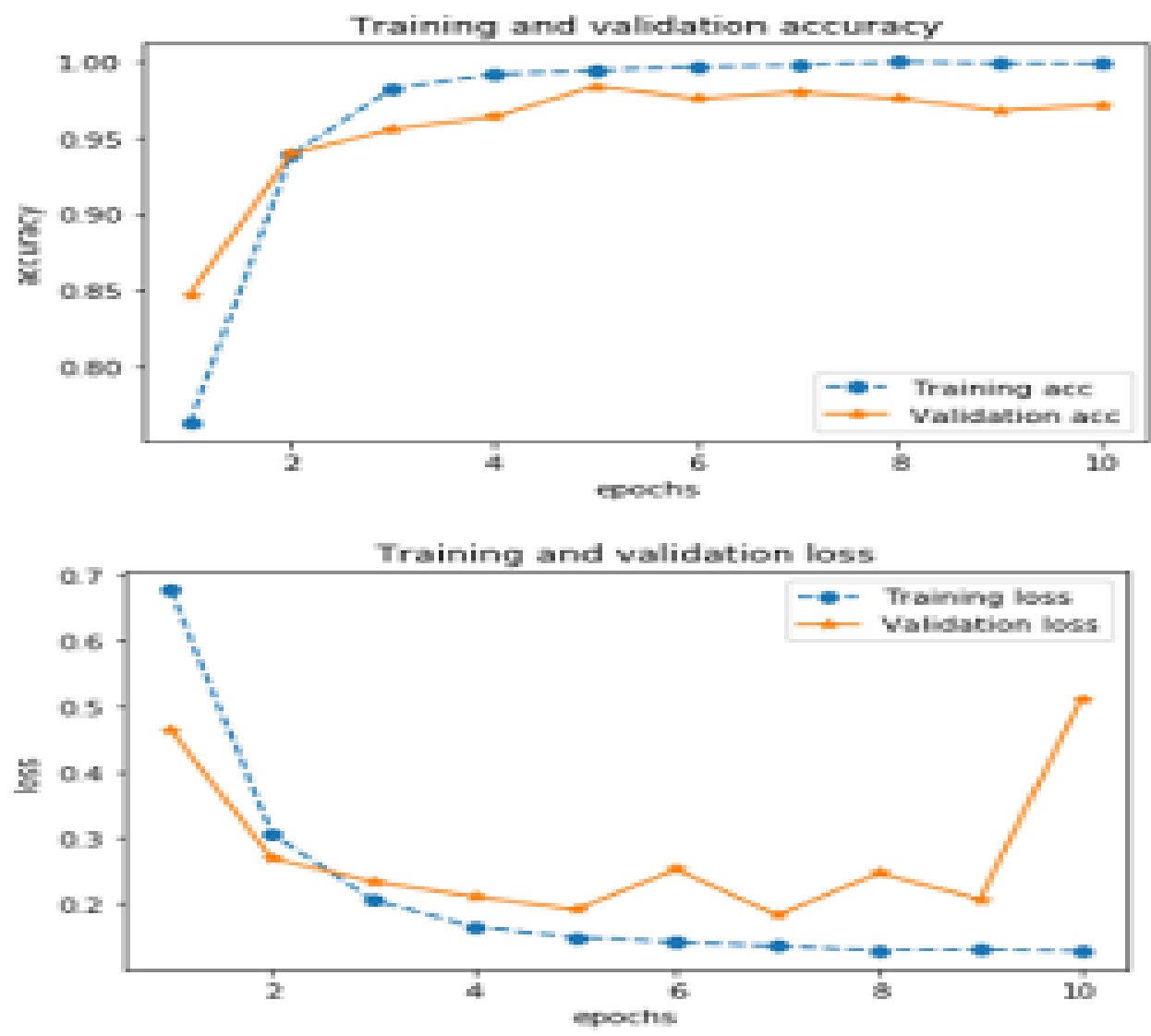

Fig 4(a): Accuracy and Loss Values after Fine-Tuning- ResNet50
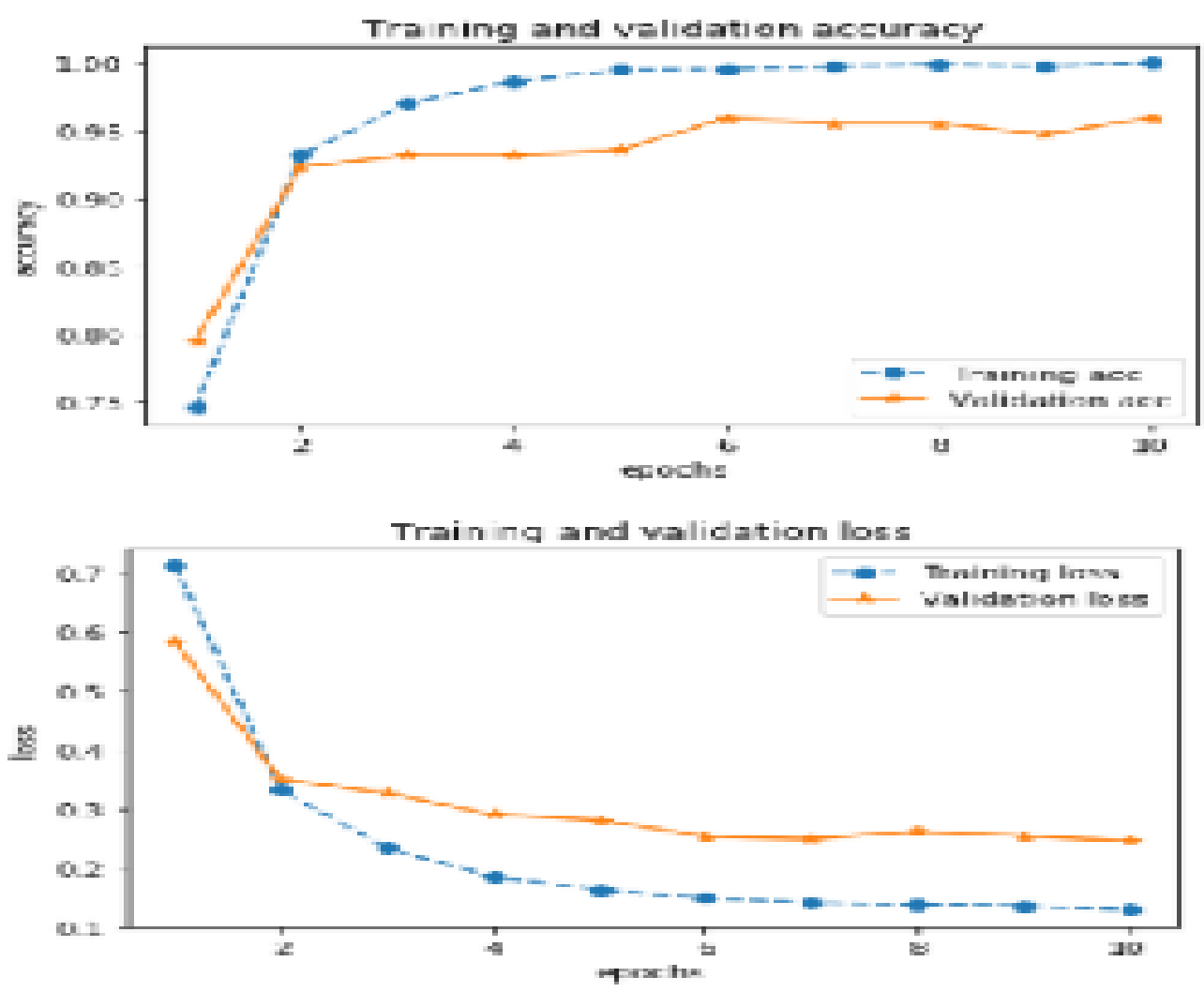

Fig 4(b): Accuracy and Loss Values after Fine-Tuning- ResNet101 

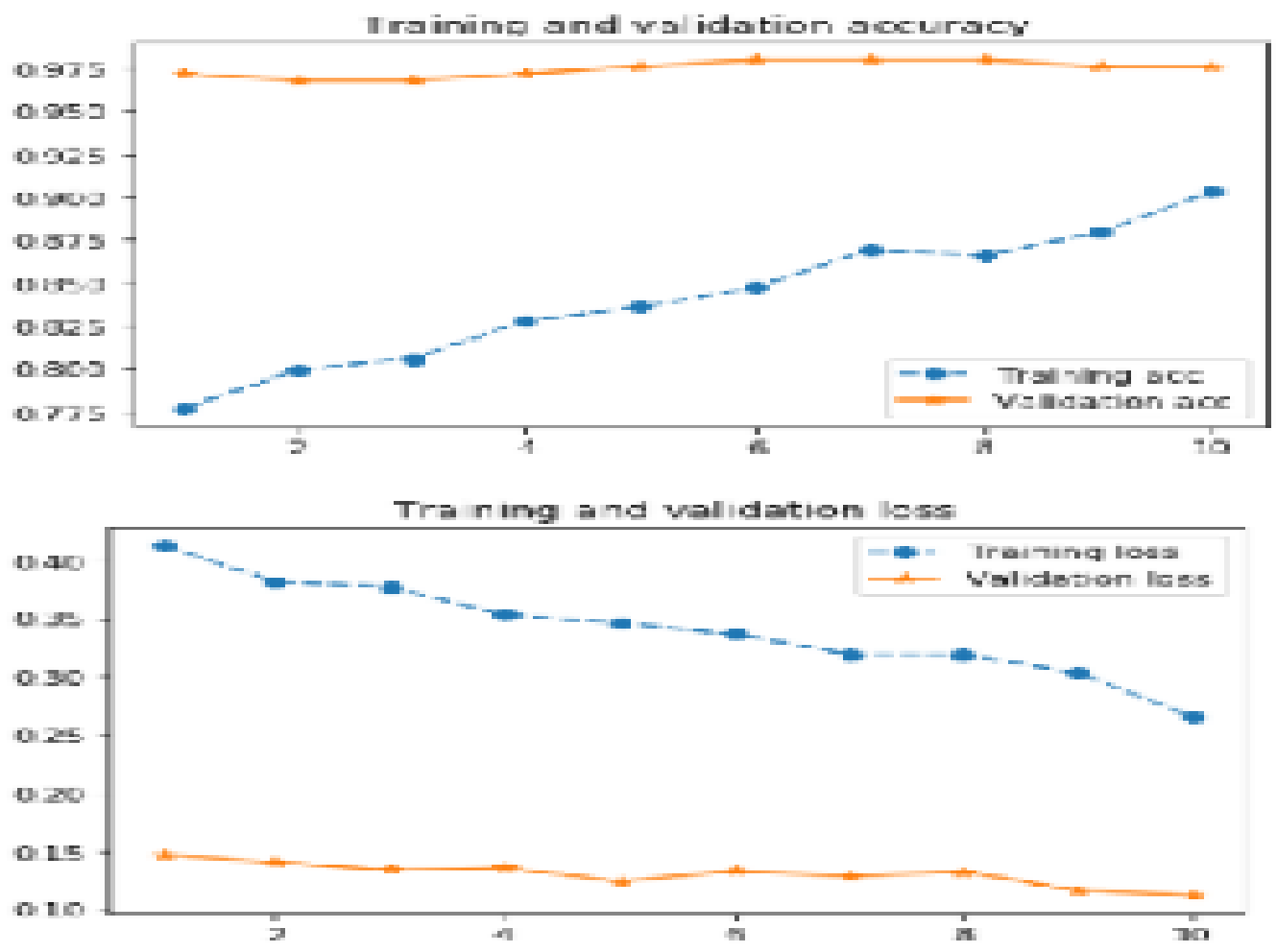

(c)

Fig 4(c): Accuracy and Loss Values after Fine- ResNet152

Table 5: Comparison with existing methods

\begin{tabular}{|l|l|c|}
\hline \multicolumn{1}{|c|}{ Method } & \multicolumn{1}{|c|}{ Dataset } & $\begin{array}{c}\text { Accuracy } \\
(\%)\end{array}$ \\
\hline $\begin{array}{l}\text { Perumal et al. } \\
{[14]}\end{array}$ & $\begin{array}{l}\text { Github open } \\
\text { repository, RSNA and } \\
\text { Google images }\end{array}$ & 93.00 \\
\hline Abbas et al. [15] & $\begin{array}{l}\text { Chest X-ray dataset } \\
{[22],[23]}\end{array}$ & 97.35 \\
\hline Gupta et al. [16] & $\begin{array}{l}\text { Chest X-ray dataset } \\
{[24],[25]}\end{array}$ & 99.52 \\
\hline $\begin{array}{l}\text { Proposed } \\
\text { ResNet152 based } \\
\text { method }\end{array}$ & $\begin{array}{l}\text { SARS-CoV-2 CT-scan } \\
\text { dataset [21] }\end{array}$ & 98.39 \\
\hline
\end{tabular}

\section{CONCLUSION}

Transfer learning is used widely in the recent years to improve the performance of deep learning neural network models. In this paper, three pre-trained deep neural network models viz., ResNet50, ResNet101 and ResNet 152 are fine-tuned to classify the COVID-19 CT lung images. The ResNet152 model achieves an accuracy of 98.39\% which is comparable to the results obtained by existing methods. The results can be further improved by fine-tuning the appropriate number of layers in the ResNet.

\section{CONFLICTS OF INTEREST}

The author declares that they have no conflicts of interest.

\section{ACKNOWLEDGMENT}

The author would like to thank Bharathiar University for the necessary support provided.

\section{REFERENCES}

[1] https://covid19.who.int/ (Accessed 8 February 2021)

[2] https://covid19.who.int/region/searo/country/in (Accessed 8 February 2021)

[3] T. Ai, Z. Yang, H. Hou, C. Zhan, C. Chen, W. Lv, Q. Tao, Z. Sun and L. Xia, "Correlation of chest CT and RT-PCR testing for coronavirus disease (COVID-19) in China: A report of 1014 cases", Radiology, Vol. 296, No. 2, 2020, pp. 532 540.

[4] A. Kovacs, P. Palasti, D. Verb, B. Bozsik, A. Palko and Z. T. Kincses, "The sensitivity and specificity of chest CT in the diagnosis of COVID-19", European Radiology, 2020, DOI: 10.1007/500330-020-07347-X.

[5] A. G. Floriano, A. F. Santiago, O. C. Nieto. and C. Y. Marquez, "A machine learning approach to medical image classification: Detecting age-related macular degeneration in fundus images", Computers and Electrical Engineering, Vol. 75,2019 , pp. $218-229$.

[6] N. Thakur and M. Juneja, "Classification of glaucoma using hybrid features with machine learning approaches", Biomedical Signal Processing and Control, Vol. 62, 102137, 2020.

[7] L. Gao and S. Wu, "Response score of deep learning for outof-distribution sample detection of medical images", Journal of Biomedical Informatics, Vol. 107, 103442, 2020.

[8] L. Mou, Y. Zhao, H. Fu, Y. Liu, J. Chang, Y. Zheng, P. Su, J. Yang and L. Chen, "CS2-Net: Deep learning segmentation of curvilinear structures in medical imaging", Medical Image Analysis, Vol. 67, 101874, 2021.

[9] K. He, X. Zhang, S. Ren and J. Sun, "Deep residual learning for image recognition", in Proceedings of 2016 IEEE 
Conference on Computer Vision and Pattern Recognition, 2016, pp. $770-778$.

[10] S. Bhattacharya, P. K. R. Maddikunta, Q. V. Pham, T. R. Gadekallu, S. Sivaramakrishnan, C. L. Chowdhary, M. Alazab and M. J. Piran, "Deep learning and medical image processing for corona virus (COVID-19) pandemic: A survey", Sustainable Cities and Society, Vol. 5, 102589 , 2021.

[11] R. Jain, M. Gupta, S. Taneja and D. J. Hemanth, "Deep learning based detection and analysis of COVID-19 on chest X-ray images", Applied Intelligence, Vol. 51, 2021, pp. 1690 $-1700$

[12] M. A. Elaziz, K. M. Hosny, A. Salah, M. M. Darwish, S. Lu and A. Sahlol, "New machine learning method for imagebased diagnosis of COVID-19", PLoS ONE, Vol. 15, No. 6, e0235187, 2020.

[13] W. Zhang, B. Pogorelsky, M. Loveland and T. Wolf, "Classification of COVID-19 X-Ray images using a combination of deep and handcrafted features", arXiv, 2101.07866, 2021.

[14] V. Perumal, V. Narayanan and S. J. S. Rajasekar, "Detection of COVID-19 using CXR and CT images using transfer learning and Haralick features", Applied Intelligence, Vol. 51, 2021, pp. $51341-51358$.

[15] A. Abbas, M. M. Abdelsamea and M. M. Gaber, "Classification of COVID-19 in chest X-ray images using DeTraC deep convolutional neural network", Applied Intelligence, Vol. 51, 2021, pp. $854-864$.

[16] A. Gupta, Anjum, S. Gupta and R. Katarya, "InstaCOvNet19: A deep learning classification model for the detection of COVID-19 patients using Chest X-ray”, Applied Soft Computing Journal, Vol. 99, 106859, 2021.

[17] A. J. Sahlol, D. Yousri, A. A. Ewees, M. A. A. Al-quanees, R. Domasevicus and M. A. Elaziz, "COVID-19 image classification using deep features and fractional-order marine predators algorithm", Scientific Reports, Vol. 10, 15364, 2020.

[18] J. D. Arias-Londono, J. A. Gomez-Garcia, L. MoroVelazquez and J. I. Godino-Llorente, "Artificial intelligence applied to chest X-ray images for the automatic detection of COVID-19: A thoughtful evaluation approach", IEEE Access, Vol. 8, 2020, pp. $226811-226827$.

[19] Q. Yang, Y. Zhang, W. Dai and S. J. Pan, "Transfer learning", Cambridge University Press, 2020.

[20] A. Fandang, "Mastering TensorFlow 1.x", Packt Publishing, 2018.

[21] E. Soares, E. Angelov, S. Biaso, M. H. Froes and D. K. Abe, "SARS-CoV-2 CT-scan dataset: A large dataset of real patients CT scans for SARS-CoV-2 identification", medRxiv, 2020. DOI:10.1101/2020.04.24.20078584.

[22] S. Candemir, S. Jaeger, K. Palaniappan, J. P. Musco, R. K. Singh, Z. Xue, A. Karargyris, S. Antani, G. Thoma, C. J. McDonald, "Lung segmentation in chest radiographs suing anatomical atlases with nonrigid registration", IEEE Transactions on Medical Imaging, Vo. 33, No. 2, 2014, pp. $577-590$.

[23] J. P. Cohen, P. Morrison and L. Dao, "COVID-19 image data collection", arXiv: 2003.11597, 2020.

[24] M. E. H. Chowdhury, T. Rahman, A. Khandakar, R. Mazhar, M. A. Kadir, Z. B. Mahbub, K. R. Islam, M. S. Khan, A. Iqbal, N. Al-Emadi, M. B. I. Reaz and T. I. Islam, "Can AI help in screening viral and COVID-19 pneumonia?" IEEE Access, arXiv, 2003.13145, 2020.

[25] G. Maguolo and L. Nanni, "A critic evaluation of methods for COVID-19 automatic detection from X-ray images, arXiv, 2004.12823, 2020

\section{ABOUT THE AUTHOR}

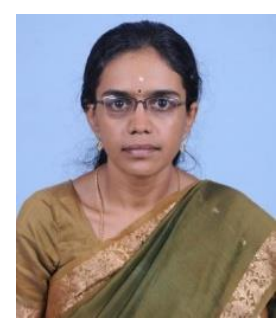

Rajeswari R. has completed her MCA in 2003 from Madurai Kamaraj University, Madurai, India and Ph.D. in Computer Science in 2012 from Bharathiar University, Coimbatore, India. She joined Department of Computer Applications, Bharathiar University, Coimbatore, India in 2005 as Assistant Professor. She is currently working as Associate Professor in the Department of Computer Applications, Bharathiar University, Coimbatore, India. She has 15 years of experience in teaching/ research. Her main research interests include medical image processing, video processing and soft computing. She is currently guiding M.Phil and Ph.D research scholars in Bharathiar University. She is a life member of Computer Society of India. 